\title{
Long chain polyunsaturated fatty acids in mothers and term babies
}

\author{
Anitha S. Kilari ${ }^{1}$, Savita S. Mehendale ${ }^{2}$, Kamini \\ D. Dangat ${ }^{1}$, Hemlata R. Yadav ${ }^{1}$, Asmita V. \\ Kulakarni', Madhavi V. Dhobale', Vaishali S. \\ Taralekar $^{2}$ and Sadhana R. Joshi ${ }^{1, *}$ \\ ${ }_{1}^{1}$ Department of Nutritional Medicine, Interactive \\ Research School for Health Affairs, Bharati Vidyapeeth \\ University, Pune 411043, India \\ ${ }^{2}$ Department of Obstetrics and Gynecology, Bharati \\ Medical College Hospital, Bharati Vidyapeeth \\ University, Pune 411043, India
}

\section{Introduction}

Long-chain polyunsaturated fatty acids (LCPUFA), especially docosahexaenoic acid (DHA) and arachidonic acid (AA), are critical nutritional sources for the developing fetus and infant [33]. DHA is the most vital LCPUFA for visual and cognitive development $[13,19]$ and is known to substantially accumulate in the brain during gestation and in the postnatal period [6]. In mothers, AA and DHA are synthesized to a limited extent, mainly in the liver by desaturation and elongation of the essential fatty acids linolenic and alpha-linolenic acid, respectively. Elongation and desaturation enzymes for LCPUFA conversion are present in the fetal liver early in gestation, but their activity appears to be low before birth [28]. Therefore, the LCPUFA that the fetus accumulates in utero are derived predominantly through placental transfer, which in turn, is influenced by maternal diet and metabolism [21]. Studies suggest that infants cannot synthesize omega 3 and omega 6 polyunsaturated fatty acids (PUFA) [19]. Human milk provides the only source of $n-6$ and $n-3$ fatty acids to support growth and development during breast-feeding and for much of early infancy [16]. Hence, the infant depends on breast milk for preformed LCPUFA in early infancy which is also influenced by maternal diet. Thus, this may compromise the maternal DHA status during lactation.

Recent studies have shown that dietary DHA intake of pregnant women between different communities is different $[11,23,24,30]$. It has also been suggested that further research into the ethnicity related differences in maternal omega 3 and omega 6 fatty acid patterns may be relevant to ethnic disparities in birth outcomes observed worldwide [29]. Further, maternal dietary intake of LCPUFA and lifestyle are known to influence the LCPUFA levels of the fetus [1,8].

Fatty acid concentrations can be assessed from both plasma and erythrocyte. It is known that fasting plasma is suitable for assessing the steady state levels of both LCPUFA following dietary intake of these and their precursors while erythrocyte fatty acids are indicative of utilization of LCPUFA into generalized membrane phospholipids. Also, the fatty acid composition of human milk is known to be influenced by maternal nutritional status, maternal dietary intakes immediately before and during gestation, and the stage of lactation [5].

Better understanding of the LCPUFA concentrations in mother and in cord blood is of importance for improving fetal DHA status not only in uncomplicated pregnancies

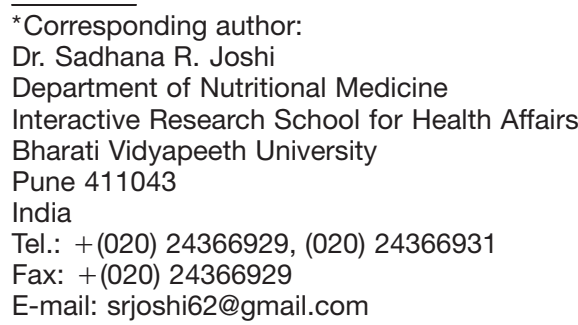


but also in disorders associated with poor DHA status, such as preeclampsia [26]. The present study describes the levels of DHA and AA in plasma, erythrocyte and breast milk simultaneously in Indian mothers who are mostly vegetarians and delivering at term. These women had a similar race, age, socio-economic status, diet and life style which collectively minimize the confounders of variability in fatty acids.

\section{Materials and methods}

The study was conducted at the Department of Obstetrics and Gynecology, Bharati Medical College Hospital, Pune during the period 2006-2007. Women with singleton pregnancies who delivered at term a baby weighing $\geq 2.5 \mathrm{~kg}$ were recruited. Pregnant women with medical problems like chronic hypertension, type 1 or type 2 diabetes mellitus, seizure disorder, alcohol or drug abuse, were excluded from the study. Women who developed preeclampsia, gestational diabetes and anemia were also excluded from the study. Delivery details and complications of each subject were recorded.

Mode of delivery in all women was recorded: $80 \%$ of deliveries were spontaneous vaginal, $12 \%$ were by cesarean section, $5 \%$ were delivered by vacuum extraction and $3 \%$ were induced. Birth outcome such as birth weight, height, head circumference and chest circumference was recorded for all neonates by standard techniques within half an hour of birth.

Maternal samples and umbilical cord samples were collected from each subject at the time of delivery but we had to omit a few hemolytic samples. This study was approved by the Institutional Ethical Committee. Written informed consent was taken from each subject. All women were of common racial origin and had similar family lifestyle.

\section{Dietary assessments}

Food frequency questionnaire was used to estimate the frequency of intake of omega three rich foods. These foods were identified using "Nutritive Values of Indian Foods" [12]. The questionnaire consisted of 17 food groups and a number of foods $(\sim 10)$ were listed under each of the food groups. The frequency of intake of foods was recorded on an 8-point scale from "never" to "thrice daily". Thus, monthly scores were calculated for each food item. For example, an item consumed once a week will have a score of four while that consumed daily will have a score of 30 .

\section{Sample collection, processing and storing}

Maternal blood samples Ten milliliter of maternal venous blood was collected into the ethylenediamine tetra-acetic acid (EDTA) vials.

Cord blood samples Ten milliliter of blood was collected into the EDTA vial from the umbilical cord just after delivery of the neonate.

All blood samples were immediately layered on histopaque (a density gradient obtained from Sigma-Aldrich) and centrifuged at $2000 \mathrm{rpm}$ for $30 \mathrm{~min}$ to separate the plasma and erythrocytes. The erythrocytes were washed 3 times with normal saline. Then the plasma and erythrocytes aliquots were stored at $-80^{\circ} \mathrm{C}$ until further analysis.

Breast milk samples Breast milk was collected on a sub-sample. At the time of breast milk sampling, the babies were 3 days of age. The mothers were instructed not to use skin care products before sampling. Breast milk samples $(3 \mathrm{~mL})$ were taken in the middle of the baby's being breast-fed and collected in a $5 \mathrm{~mL}$ vial, stored at $-80^{\circ} \mathrm{C}$, and analyzed within 10 days.

Fatty acid analysis Plasma and erythrocytes methyl esters were prepared by the method of Manku et al. [25, 26]. Briefly, transesterification of the phospholipid fraction was carried out using hydrochloric acid-methanol. These were separated using a Perkin Elmer gas chromatograph (SP 2330, 30 m capillary Supelco column). Helium was used as carrier gas at $1 \mathrm{~mL} / \mathrm{min}$. Oven temperature was held at $150^{\circ} \mathrm{C}$ for $10 \mathrm{~min}$, programmed to rise from 150 to $220^{\circ} \mathrm{C}$ at $10^{\circ} \mathrm{C} / \mathrm{min}$, and at $220^{\circ} \mathrm{C}$ for $10 \mathrm{~min}$. The detector temperature was $275^{\circ} \mathrm{C}$ and the injector temperature was $240^{\circ} \mathrm{C}$. Retention times and peak areas were automatically computed. Peaks were identified by comparison with standard fatty acid methyl esters (Sigma Aldrich, Inc. St. Louis, USA). Fatty acids were expressed as $\mathrm{g} / 100 \mathrm{~g}$ fatty acid. The total omega 3 fatty acids included alpha linolenic acid, eicosapentaenoic acid and DHA while total omega 6 fatty acids included linolenic acid, gamma linolenic acid, di-homo-gamma linolenic acid, docosapentaenoic acid and AA.

Statistical analysis Values were presented as mean \pm SD. The data were analyzed using SPSS/PC + package (Version 11.0, Chicago, IL). Skewed variables (maternal arachidonic acid and docosahexaenoic acid in both plasma and erythrocyte, cord plasma docosahexaenoic acid and breast milk docosahexaenoic acid and arachidonic acid) were log transformed. Mean values of the estimates of various parameters for maternal and cord samples were compared using the Student $t$-test at conventional levels of significance. Correlation between variables was studied using Pearson's correlation analysis.

\section{Results}

Table 1 shows the characteristics of the mothers and neonates. All women consumed green leafy vegetables which is mainly rich in alpha linolenic acid, a precursor of DHA for about 4-5 times a week.

Table 2 shows the maternal (both plasma and erythrocyte), cord (both plasma and erythrocyte) and breast milk fatty acid composition. The maternal plasma omega 6 /omega 3 fatty acid ratio was $20.5 \pm 7.9$ whereas it was $7.33 \pm 2.38$ in erythrocytes. Similarly, omega $6 /$ omega 3 fatty acids ratio was $10.6 \pm 3.54$ in cord plasma and $5.31 \pm 1.2$ in erythrocytes.

Cord levels of both plasma and erythrocyte DHA and AA were higher than the maternal blood levels $(P<0.001)$.

There was a positive weak association between maternal plasma DHA and maternal erythrocyte $\mathrm{DHA}(\mathrm{n}=121$, $r=0.492, P=0.001$ ) and also between maternal plasma 
AA and maternal erythrocyte AA $(n=121, r=0.242$, $P=0.007$ ) (Figure 1). This association was seen in cord samples only for DHA $(n=115, r=0.501, P=0.001)$ but not for AA.

There was also a weak positive association between maternal plasma DHA and cord plasma DHA $(n=105$, $r=0.337, P=0.001$ ); maternal erythrocyte DHA and cord erythrocyte DHA $(n=84, r=0.290, P=0.007)$ (Figure 2) whereas this association was not seen for both plasma and erythrocyte AA concentrations between mother and cord.

Maternal plasma DHA and AA concentrations did not show any associations with milk DHA and AA concentrations. However, maternal plasma omega 3 fatty acids were positively associated with milk omega $3(n=81$, $r=0.230, P=0.039$ ). Further, maternal plasma omega 6 fatty acids were also associated with milk omega 6 fatty acids $(n=81, r=0.280, P=0.011)$ (Figure 3$)$.

\section{Discussion}

We simultaneously examined LCPUFA status in plasma as well as in erythrocytes of maternal and cord blood along with breast milk samples in mothers of term babies weighing $\geq 2.5 \mathrm{~kg}$. Our results indicate 1) Plasma and erythrocyte DHA and AA levels were higher in cord blood as compared to maternal blood. 2) Maternal plasma DHA and $A A$ were positively associated with maternal erythrocyte $\mathrm{DHA}$ and $\mathrm{AA}$, respectively. 3) Maternal plasma and erythrocyte DHA levels were associated with cord plasma and erythrocyte DHA levels but no association was seen with AA. 4) Maternal plasma omega 3 and omega 6 fatty acids were positively associated with milk omega 3 and omega 6 fatty acids, respectively.

Maternal erythrocyte DHA levels were similar and AA was lower in our study than those reported in a UK cohort [27]. However, both AA and DHA levels in our

Table 1 Maternal and neonatal characteristics.

\begin{tabular}{lc}
\hline $\begin{array}{l}\text { Maternal characteristics } \\
(\mathrm{n}=148)\end{array}$ & Mean $\pm \mathrm{SD}$ \\
\hline Age (years) & $22.5 \pm 3$ \\
Income per month $(\$)$ & $89.2 \pm 48.8$ \\
Education (grade) & $10 \pm 3$ \\
Total gestation (weeks) & $39 \pm 0.9$ \\
Systolic blood pressure $(\mathrm{mm} \mathrm{Hg})$ & $125.4 \pm 9.8$ \\
Diastolic blood pressure $(\mathrm{mm} \mathrm{Hg})$ & $79.9 \pm 6.9$ \\
Pulse & $83.2 \pm 6$ \\
Weight $(\mathrm{kg})$ & $50.4 \pm 7.6$ \\
Height $(\mathrm{cm})$ & $151.7 \pm 5.4$ \\
Gravida & $1.7 \pm 1$ \\
Neonatal characteristics & \\
$\quad$ Baby weight $(\mathrm{kg})$ & $2.9 \pm 0.3$ \\
Baby length $(\mathrm{cm})$ & $48.4 \pm 2.5$ \\
Head circumference $(\mathrm{cm})$ & $34.5 \pm 1.8$ \\
Chest circumference $(\mathrm{cm})$ & $32.6 \pm 1.4$ \\
\hline
\end{tabular}

cohort were almost twice lower than those reported in the United States and European women [2, 32]. One of the difficulties in defining optimal intake of omega 3 fatty acids is the lack of information on biochemical markers of omega 3 fatty acid status. Most studies estimate the intakes of omega 3 fatty acids by food recall and food frequency questionnaire using food composition tables which are an indirect measure of omega 3 fatty acid status [7]. Our results suggest that levels of LCPUFA vary between different communities and may be dependent on their intakes and lifestyle. Thus, our study could be useful to understand the requirement of essential fatty acids in Indian women delivering at term.

Further, our results showed that maternal plasma as well as erythrocyte DHA and $A A$ concentrations were higher in cord as compared to maternal blood. This suggests that large quantities of maternal DHA and $A A$ are diverted to the fetus since they are important for fetal development. DHA was preferentially transferred from the maternal to the fetal circulation compared to other PUFAs [22]. This is consistent with numerous studies that showed higher LCPUFA concentrations in fetal than in maternal circulation [3, 14, 15] and suggests a preferential uptake of LCPUFA by the placenta.

Our results showed a positive association between both plasma and erythrocyte maternal DHA and cord

Table 2 Maternal, cord and milk fatty acid levels ( $\mathrm{g} / 100 \mathrm{~g}$ fatty acids).

\begin{tabular}{|c|c|c|}
\hline & $\begin{array}{l}\text { Plasma } \\
(n=148) \\
\text { Mean } \pm \text { SD }\end{array}$ & $\begin{array}{l}\text { Erythrocyte } \\
(\mathrm{n}=121) \\
\text { Mean } \pm \text { SD }\end{array}$ \\
\hline \multicolumn{3}{|l|}{ Maternal fatty acids } \\
\hline AA [20:4 (n-6)] & $6.3 \pm 2.1$ & $13.4 \pm 2.4$ \\
\hline DHA [22:6 (n-3)] & $1.3 \pm 0.5$ & $3.5 \pm 1$ \\
\hline Total omega 3 & $2.1 \pm 0.7$ & $3.9 \pm 1.1$ \\
\hline Total omega 6 & $39.1 \pm 6$ & $26.4 \pm 3.1$ \\
\hline \multirow[t]{2}{*}{$\mathrm{AA}: \mathrm{DHA}$} & $5.1 \pm 1.8$ & $4.2 \pm 1.4$ \\
\hline & $\begin{array}{l}\text { Plasma } \\
(n=132)\end{array}$ & $\begin{array}{l}\text { Erythrocyte } \\
(n=117)\end{array}$ \\
\hline \multicolumn{3}{|l|}{ Cord fatty acids } \\
\hline AA [20:4 (n-6)] & $13.6 \pm 3.9^{\star}$ & $17.2 \pm 2^{*}$ \\
\hline DHA [22:6 (n-3)] & $2.1 \pm 0.8^{*}$ & $4.2 \pm 0.9^{*}$ \\
\hline Total omega 3 & $2.9 \pm 0.9^{*}$ & $4.8 \pm 1^{*}$ \\
\hline Total omega 6 & $28.8 \pm 6.2^{*}$ & $24.2 \pm 2.5^{\star}$ \\
\hline \multirow[t]{2}{*}{ AA:DHA } & $7.2 \pm 2.7^{*}$ & $4.3 \pm 0.9$ \\
\hline & $\begin{array}{l}\text { Breast milk } \\
(\mathrm{n}=89)\end{array}$ & \\
\hline \multicolumn{3}{|l|}{ Fatty acids } \\
\hline AA [20:4 (n-6)] & $1 \pm 0.5$ & \\
\hline $\mathrm{DHA}[22: 6(n-3)]$ & $0.2 \pm 0.1$ & \\
\hline Total omega 3 & $1.2 \pm 0.4$ & \\
\hline Total omega 6 & $24 \pm 4.5$ & \\
\hline $\mathrm{AA}: \mathrm{DHA}$ & $5.3 \pm 3$ & \\
\hline
\end{tabular}

$\mathrm{AA}=$ arachidonic acid, $\mathrm{DHA}=$ docosahexaenoic acid. ${ }^{*} \mathrm{P}<0.001$. 

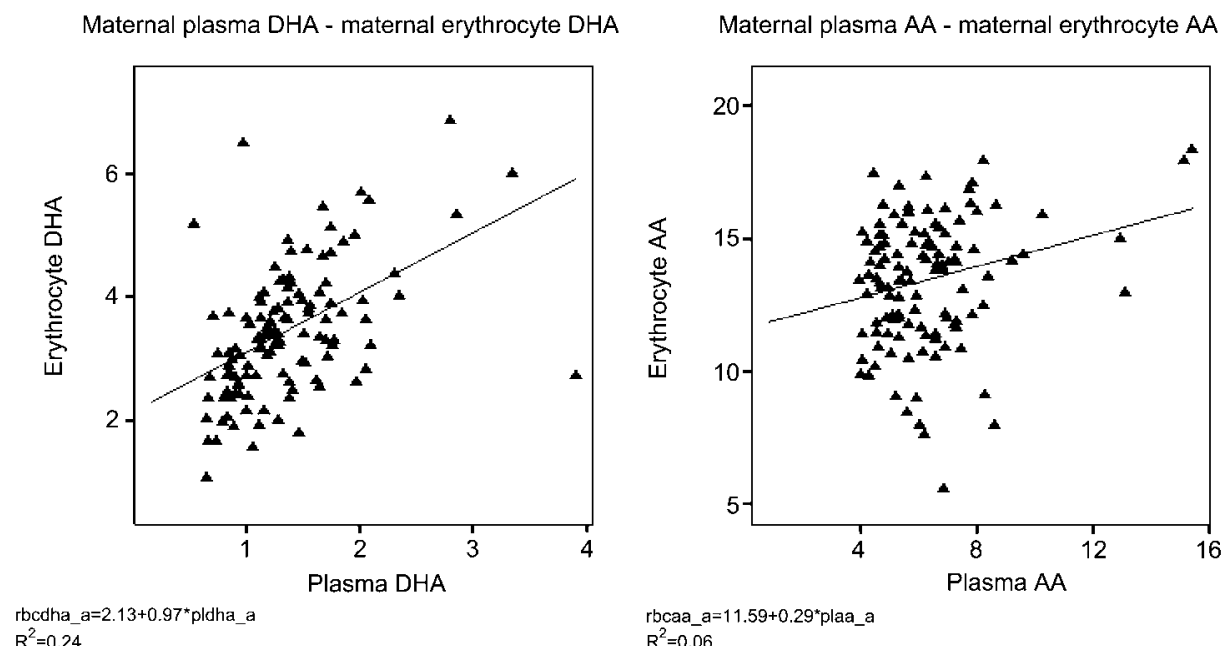

Figure 1 Maternal plasma docosahexaenoic acid $(\mathrm{DHA})$ and arachidonic acid $(\mathrm{AA})$ with maternal erythrocyte concentrations.
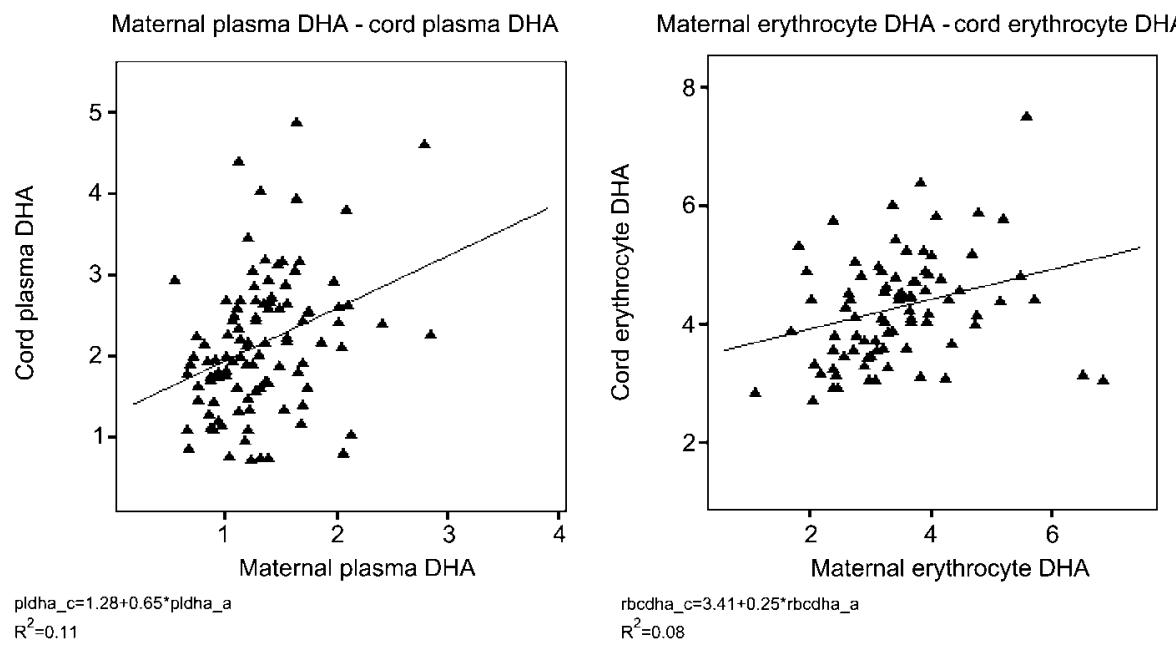

Figure 2 Associations between maternal docosahexaenoic acid (DHA) (plasma and erythrocytes) with cord concentrations.
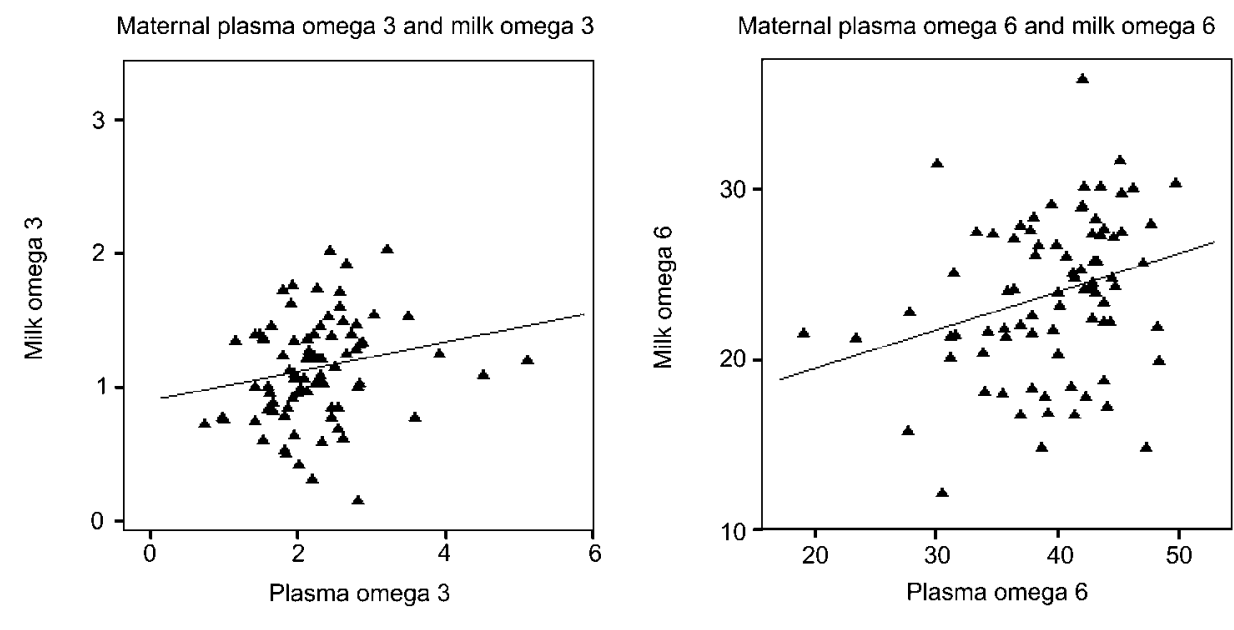

$\min 3=0.89+0.11^{*} \mathrm{pln} 3 \_$a

miln $6=14.98+0.22^{*} \mathrm{pln} 6 \_$a

$\mathrm{R}^{2}=0.04$

$\min 6=14.98$
$\mathrm{R}^{2}=0.08$

Figure 3 Associations between omega 3 and omega 6 fatty acids in maternal plasma and milk. 
blood DHA. This association was not seen with AA (both plasma and erythrocyte). Studies have shown that because of limited fetal capacity to synthesize LCPUFA, the fetus depends on LCPUFA transfer across the placenta. Although molecular mechanisms of placental LCPUFA uptake and transport are not fully understood, it has been demonstrated that a preferential DHA transfer exists, and is supported by our data. Thus, the placenta plays a vital role for the selective channeling of DHA from maternal diet and body stores to the fetus [9, 13]. Further studies would help to understand the molecular mechanisms of LCPUFA transport across the placenta and the impact on pregnancy outcome and fetal development.

In our study, maternal plasma DHA or AA did not show a direct association with milk DHA or AA. However, there was a positive association between plasma omega 3 fatty acid concentrations with milk omega 3 fatty acid concentrations. A similar trend was also seen in the case of omega 6 fatty acid concentrations. This might indicate that the mammary gland also plays a role in determining the DHA status of milk. Thus, milk LCPUFA reflects the concentrations of LCPUFA in maternal plasma which, in turn, are dependent on maternal diet [17] and probably, maternal and mammary gland activities of the desaturases and elongases involved in converting dietary linolenic acid and alpha linolenic acid to LCPUFA. A recent study has reported that the combined consumption of $A A$ and omega 3 LCPUFA showed a positive correlation between milk AA and DHA concentrations with their corresponding values in erythrocyte phospholipids [31].

In our cohort, comprising mainly of consumers of a vegetarian diet, the mean levels of milk DHA are $0.2 \mathrm{~g} /$ $100 \mathrm{~g}$ total fatty acids. This is consistent with 0.2-0.3 levels of DHA in milk from women in Canada, the USA, Europe and Australia $[16,18]$. In contrast, the DHA of human colostrums was $0.6 \mathrm{~g} / 100 \mathrm{~g}$ in Australian than in European samples [10]. The levels of DHA in human milk are known to vary widely within and among populations and women consuming diets high in fish and other marine animals often have $>1 \mathrm{~g} \mathrm{DHA} / 100 \mathrm{~g}$ total fatty acids in breast milk [16]. High contents of DHA and total n-3 LCPUFA were observed in the colostrum of St. Lucian women who consume abundant fish [10]. Human milk contains DHA in varying amounts depending on maternal diet [22].

During pregnancy, maternal LCPUFA body stores more than the actual diet, influence the LCPUFA status of breast milk fatty acid composition [20]. A recent review strongly supports the benefits of adding DHA and AA to infant formula and also advises to continue the provision of dietary sources of LCPUFA during the second 6 months of life [19]. Our results suggest that improved maternal LCPUFA status would greatly improve the milk LCPUFA status which ultimately would improve the neurodevelopment of the infant. A recent study in central Europe has shown that a dose of $200 \mathrm{mg} /$ day DHA from mid-pregnancy through lactation is appropriate to improve the DHA status of mothers and infants [4].

Our findings have important implications for the effects of omega 3 fatty acid interventions in pregnancy and lactation that support improved fetal growth, pregnancy outcomes and postnatal development.

\section{References}

[1] Agostoni C, Galli C, Riva E, Colombo C, Giovannini M, Marangoni F. Reduced docosahexaenoic acid synthesis may contribute to growth restriction in infants born to mothers who smoke. J Pediatr. 2005;147:854-6.

[2] Al MD, van Houwelingen AC, Hornstra G. Long-chain polyunsaturated fatty acids, pregnancy, and pregnancy outcome. Am J Clin Nutr. 2000;71:285S-91S.

[3] Al MD, Hornstra G, van der schouw YT, Bulsra-Ramakers MT, Huisjes HJ. Biochemical EFA status of mothers and their neonates after normal pregnancy. Early Human Dev. 1990;24:239-48.

[4] Bergmann RL, Haschke-Becher E, Klassen-Wigger P, Bergmann KE, Richter R, Dudenhausen JW, et al. Supplementation with $200 \mathrm{mg} /$ day docosahexaenoic acid from mid-pregnancy through lactation improves the docosahexaenoic acid status of mothers with a habitually low fish intake and of their infants. Ann Nutr Metab. 2008;52: 157-66.

[5] Bokor S, Koletzko B, Decsi T. Systematic review of fatty acid composition of human milk from mothers of preterm compared to full-term infants. Ann Nutr Metab. 2007; 51:550-6.

[6] Cetin I, Koletzko B. Long-chain omega-3 fatty acid supply in pregnancy and lactation. Curr Opin Clin Nutr Metab Care. 2008;11:297-302.

[7] Denomme J, Stark KD, Holub BJ. Directly quantitated dietary $(n-3)$ fatty acid intakes of pregnant Canadian women are lower than current dietary recommendations. The American Society for Nutritional Sciences. J Nutr. 2005; 135:206-11.

[8] Duttaroy AK. Transport of fatty acids across the human placenta: a review. Prog Lipid Res. 2009;48:52-61.

[9] Dutta-Roy AK. Transport mechanisms for long-chain polyunsaturated fatty acids in the human placenta. Am J Clin Nutr. 2000;71:315S-22S.

[10] Fidler N, Koletzko B. The fatty acid composition of human colostrum. Eur J Nutr. 2000;39:31-7.

[11] Franke C, Verwied-Jorky S, Campoy C, Trak-Fellermeier M, Decsi T, Dolz V, et al. Dietary intake of natural sources of docosahexaenoic acid and folate in pregnant women of three european cohorts. Ann Nutr Metab. 2008;53:167-74.

[12] Ghafoorunissa and Jyotsna Pangrekar. Vegetables as sources of $\alpha$-linolenic acid in Indian diets. Food Chemistry. 1993;47:121-4.

[13] Hanebutt FL, Demmelmair H, SchiessI B, Larqué E, Koletzko B. Long-chain polyunsaturated fatty acid (LC-PUFA) transfer across the placenta. Clin Nutr. 2008;27:685-93.

[14] Innis SM. Essential fatty acids in growth and development. Prog Lipid Res. 1986;30:39-103.

[15] Innis SM. Polyunsaturated fatty acids in human milk: an essential role in infant development. Adv Exp Med Biol. 2004;554:27-43. 
[16] Innis SM. Human milk: maternal dietary lipids and infant development. Proc Nutr Soc. 2007;66:397-404.

[17] Jensen RG, Lammi-Keefe CJ, Henderson RA, Bush VJ, Ferris AM. Effect of dietary intake of $n-6$ and $n-3$ fatty acids on the fatty acid composition of human milk in North America. J Pediatr. 1992;120:S87-92.

[18] Jensen CL, Voigt RG, Prager TC, Zou YL, Fraley JK, Rozelle JC, et al. Effects of maternal docosahexaenoic acid intake on visual function and neurodevelopment in breastfed term infants. Am J Clin Nutr. 2005;82:125-32.

[19] Koletzko B, Lien E, Agostoni C, Böhles H, Campoy C, Cetin I, et al. The roles of long-chain polyunsaturated fatty acids in pregnancy, lactation and infancy: review of current knowledge and consensus recommendations. J Perinat Med. 2008;36:5-14.

[20] Koletzko B, Rodriguez-Palmero M, Demmelmair H, Fidler $\mathrm{N}$, Jensen R, Sauerwald T. Physiological aspects of human milk lipids. Early Hum Dev. 2001;65:S3-18.

[21] Krauss-Etschmann S, Shadid R, Campoy C, Hoster E, Demmelmair $H$, Jiménez $M$, et al. Effects of fish-oil and folate supplementation of pregnant women on maternal and fetal plasma concentrations of docosahexaenoic acid and eicosapentaenoic acid: a European randomized multicenter trial. Fish oil and folate supplementation of pregnant women and maternal and fetal DHA and EPA plasma levels - a randomized European multicenter trial. Am J Clin Nutr. 2007;85:1392-400.

[22] Lauritzen L, Hansen HS, Jørgensen MH, Michaelsen KF. The essentiality of long chain $n-3$ fatty acids in relation to development and function of the brain and retina. Prog Lipid Res. 2001;40:1-94.

[23] Linseisen J, Kesse E, Slimani N, Bueno-De-Mesquita HB, Ocké MC, Skeie G, et al. Meat consumption in the European Prospective Investigation into Cancer and Nutrition (EPIC) cohorts: results from 24-hour dietary recalls. Public Health Nutr. 2002(a);5:1243-58.

[24] Linseisen J, Bergström E, Gafá L, González CA, Thiébaut A, Trichopoulou A, et al. Consumption of added fats and oils in the European Prospective Investigation into Cancer and Nutrition (EPIC) centres across 10 European countries as assessed by 24 -hour dietary recalls. Public Health Nutr. 2002(b);5:1227-42.
[25] Manku MS, Horrobin DF, Huang S, Morse N. Fatty acids in plasma and red cell membranes in normal humans. Lipids. 1983;18:906-8.

[26] Mehendale S, Kilari A, Dangat K, Taralekar V, Mahadik S, Joshi S. Fatty acids, antioxidants, and oxidative stress in pre-eclampsia. Int J Gynaecol Obstet. 2008;100:234-8.

[27] Stewart F, Rodie VA, Ramsay JE, Greer IA, Freeman DJ, Meyer BJ. Longitudinal assessment of erythrocyte fatty acid composition throughout pregnancy and post partum. Lipids. 2007;42:335-44.

[28] Uauy R, Mena P, Wegher B, Nieto S, Salem N Jr. Long chain polyunsaturated fatty acid formation in neonates: effect of gestational age and intrauterine growth. Pediatr Res. 2000;47:127-35.

[29] Van Eijsden M, Hornstra G, van der Wal MF, Bonsel GJ. Ethnic differences in early pregnancy maternal n-3 and n6 fatty acid concentrations: an explorative analysis. $\mathrm{Br} \mathrm{J}$ Nutr. 2008;5:1-8.

[30] Welch AA, Lund E, Amiano P, Dorronsoro M, Brustad M, Kumle $\mathrm{M}$, et al. Variability of fish consumption within the 10 European countries participating in the European Investigation into Cancer and Nutrition (EPIC) study. Public Health Nutr. 2002;5:1273-85.

[31] Weseler AR, Dirix CE, Bruins MJ, Hornstra G. Dietary arachidonic acid dose-dependently increases the arachidonic acid concentration in human milk. J Nutr. 2008;138: 2190-7.

[32] Wijendran V, Bendel RB, Couch SC, Philipson EH, Thomsen K, Zhang X, et al. Maternal plasma phospholipid polyunsaturated fatty acids in pregnancy with and without gestational diabetes mellitus: relations with maternal factors. Am J Clin Nutr. 1999;70:53-61.

[33] Xu Y, Wang Q, Cook TJ, Knipp GT. Effect of placental fatty acid metabolism and regulation by peroxisome proliferator activated receptor on pregnancy and fetal outcomes. $J$ Pharm Sci. 2007;96:2582-6.

The authors stated that there are no conflicts of interest regarding the publication of this article.

Received December 18, 2008. Revised February 25, 2009. Accepted March 5, 2009. Previously published online June 3, 2009. 